\title{
Accurate Determination of Malachite Green and Leucomalachite Green in Fish using Isotope Dilution Liquid Chromatography/Mass Spectrometry (ID-LC/MS)
}

\author{
Seonghee Ahn, ${ }^{\dagger, *}$ Byungjoo Kim, ${ }^{\dagger}$ Yunjung Lee, ${ }^{\dagger,+, a}$ and Jeongkwon Kim \\ ${ }^{\dagger}$ Division of Metrology for Quality Life, Korea Research Institute of Standards and Science, Yuseong, Daejon 305-600, Korea \\ ${ }^{*}$ E-mail: sahn@kriss.re.kr \\ ${ }^{\ddagger}$ Departments of Chemistry, Chungnam University, Yuseong, Daejon 305-764, Korea \\ Received May 28, 2010, Accepted September 8, 2010
}

\begin{abstract}
Malachite green (MG) has been used world-widely in aquaculture as a parasiticide or fungicide. Although MG performed successfully, it has not been permitted for use in aquaculture from European Union, USA, and Canada because of its carcinogenicity and mutagenicity. We developed a sensitive and specific method to determine MG and its principal metabolite, leucomalachite green (LMG), respectively by isotope dilution liquid chromatography mass spectrometry (ID-LC/MS). To enhance the extraction recovery of MG and LMG from fish tissue, an additional step, saponification, was introduced in sample preparation process to remove fat in sample extract, which hampered the performance of SPE columns. The residue of MG and LMG in fish was analyzed using liquid chromatography mass spectrometry in the selected ion monitoring (SIM) mode by monitoring at $\mathrm{m} / z 329$ and 334 for MG and $d_{5}$-MG and at $m / z 331$ and 337 for $\mathrm{LMG}$ and ${ }^{13} \mathrm{C}_{6}$-LMG, respectively. This method was validated by comparing with the value of the reference material provided by Laboratory Government Chemistry (LGC). The results agreed within the measurement uncertainty and the accuracy was much improved than the provided reference value by LGC.
\end{abstract}

Key Words: Malachite green, Leucomalachite green, Isotope dilution mass spectrometry, Saponification, LC/MS

\section{Introduction}

Malachite green (MG) is a triphenylmethane dye that has been used traditionally in aquaculture to treat fish eggs and adult fish for fungal infections and ectoparasites since $1930 .{ }^{1,2}$ Eighty percentage or more of absorbed MG is transformed metabolically to its reduced form, leucomalachite green (LMG). ${ }^{3}$ The chemical structures of MG and LMG are shown in Scheme 1. The powerful antimicrobial activity of MG has been attributed to inhibition of intracellular enzymes, intercalation into DNA, and/or interaction with cellular membranes. ${ }^{4,5} \mathrm{MG}$ is related structurally to other carcinogenic triphenylmethane dyes: gentian violet, a thyroid and liver carcinogen in rodents, and pararoaniline, a bladder carcinogen in humans. ${ }^{6,7}$ Hence, the use of MG has been prohibited in Europe, United States or Canada due to its suspected carcinogenic properties. The European

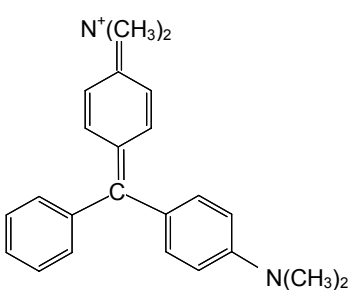

Malachite green

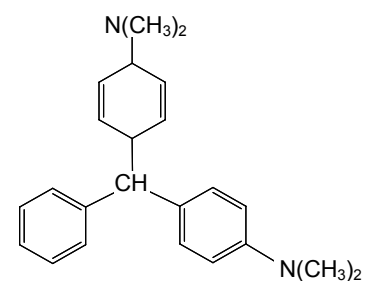

Leucomalachite green
Scheme 1. Chemical structures of malachite green and leucomalachite green.

${ }^{a}$ Current address: COSMAX, Hwaseong-si, Gyeonggi-do 902, Korea
Commission requires that the minimum required performance limit for the analysis of MG in fish products was set at $2 \mu \mathrm{g} / \mathrm{kg}$ as the sum of MG and LMG. ${ }^{8}$ Furthermore, it has been reported that $\mathrm{LMG}$ causes a greater number of and more severe changes of DNA than MG does. . LMG is also a target analyte of monitoring $\mathrm{MG}$ abuse because most of absorbed MG is reduced as LMG and retained as long as 40 days in fish which is much longer than MG did. ${ }^{2,10,11}$ Hence, it is much necessary for determination of LMG sensitively and accurately as well as MG.

Various analytical methods have been reported for the analysis of MG and LMG residue. Specially, UV/VIS light detection is working greatly with HPLC for the green color of MG. However, colorless LMG cannot be detected with an UV/VIS detector. This problem has been resolved with either pre- or post-column oxidation of LMG to MG, allowing the total amount of MG residue. ${ }^{12-15}$ However, the oxidation column causes problems including rapid depletion and peak broadening, which lead to decrease in method sensitivity. Recently, detection with mass spectrometric techniques has been proved to be more sensitive and specific than spectroscopic detection. Hence, the detection of MG and LMG by mass spectrometry without the oxidation step was accomplished. ${ }^{16-19}$ The other techniques using GC-MS, ${ }^{20,21}$ electrochemical detection, ${ }^{22}$ and spectrometry $^{23,24}$ have been achieved also.

In this study, an experimental approach to improve accuracy for determination of MG and LMG in fish tissue has been developed using isotope dilution liquid chromatography mass spectrometry (ID-LC/MS). Isotopic analogues of both MG and LMG were spiked to fish tissue sample as internal standards, and then the extract was obtained through the liquid-liquid extraction process and two stages of SPE clean-up. In sample 
preparation process, the additional defatting step by saponification is employed to improve the overall extraction recovery of MG and LMG. Generally, there are some approaches to remove co-eluted fat from fish or meat such as liquid-liquid partitioning using hexane or acetone, ${ }^{25}$ soxhlet extraction, ${ }^{26}$ freezing-lipid filtration ${ }^{27}$ and acid ${ }^{28}$ or basic treatment like saponification. However, liquid-liquid partitioning and soxhlet extraction are time-consuming and labor-intensive. Even though freezing-lipid filtration is a simple method for defatting, it is not easy to control the temperature to keep the lipid to freeze during sample manipulation. Therefore, we chose saponification with basic treatment because it was simple and completely removed fat. This proposed method was evaluated by analysis of two other kinds of fishes fortified with MG and LMG at various levels. Additionally, it was validated by comparing with the value of the reference material provided by Laboratory of Government Chemistry (UK).

\section{Experimental}

Materials. Malachite green and leucomalachite green was purchased from Aldrich (Milwaukee, WI, USA) and was used as a primary reference material without further purification. Purity of MG is $95 \%$ and LMG is $97 \%$ as provided by manufacture's analysis certificate. Deuterated malachite green $\left(d_{5}-\mathrm{MG}\right)$ was obtained from Witega (Berlin, Germany) and ${ }^{13} \mathrm{C}_{6}$-leucomalachite green from Cambridge Isotope Laboratories (Andover, MA, USA). HPLC grade organic solvents, methanol, acetonitrile and dichloromethane, were manufactured by Burdick and Jackson (Muskegon, MI, USA). Sodium hydroxide, hydroxylamine hydrochloride (HAH), $p$-toluenesulfonic acid, diethylene glycol and ammonium acetate were purchased from Aldrich (Milwaukee, WI, USA). Ascorbic acid was obtained from Junsei (Tokyo, Japan) and alumina from Selecto Scientific (Suwanee, GA, USA).

Standard solution. MG and LMG standard solutions were prepared and verified in our laboratory. The procedure for gravimetric preparation and verification was described elsewhere. $^{29,30}$ The brief description of the procedure is as following. Four replicates of MG stock solution $(2 \mathrm{mg} / \mathrm{kg})$ were gravimetrically prepared in acetonitrile with $1 \%$ acetic acid and four replicates of LMG stock solution $(2 \mathrm{mg} / \mathrm{kg}$ ) were prepared in acetonitrile containing ascorbic acid $(10 \mu \mathrm{g} / \mathrm{mL})$ for their stability. A $d_{5}-\mathrm{MG}$ and a ${ }^{13} \mathrm{C}_{6}-\mathrm{LMG}$ solution were prepared in the same ways with the corresponding native compounds. For MG and LMG, two isotope ratio standard solutions for each of four stock solutions were prepared by gravimetrically mixing with the corresponding isotope labeled standard solution as 1:1 isotope ratio, respectively. We tested self-consistency within and among the standard solutions by inter-comparing the eight isotope ratio standards by LC/MS. Based on their LC/MS results, repeatability of preparing standard solutions and isotope ratio standards were evaluated and then, one isotope ratio standard for each of MG and LMG was selected and used for the calibration and further sample analysis.

Sample preparation. For method development, blank fish samples, salmon and tuna, were purchased from a local market. They were cut into small pieces and blended until contents were uniformed. These blended samples were bottled and stored below $-20{ }^{\circ} \mathrm{C}$ until analyzed. Before subsampling, the frozen fish sample was placed at room temperature for 1 hour, which was still covered with ice on the surface. Then, it was gravimetrically fortified with MG and LMG. Method validation was performed with incurred salmon which was supplied from LGC. It was distributed as a blended shape and stored below $-20{ }^{\circ} \mathrm{C}$ in the dark. Two grams of sample (either fortified one or incurred one) was accurately weighed into individual $50-\mathrm{mL}$ Teflon centrifuge tubes and spiked with appropriate amounts of the $d_{5}$-MG and ${ }^{13} \mathrm{C}_{6}$-LMG standard solutions to make $1: 1$ isotope ratio. Sample extraction and clean-up processes are basically similar to methods reported in literatures, ${ }^{30,31}$ but an important modification was done to increase overall recovery of target analytes. The sample was vortexing for $2 \mathrm{~min}$ with $5 \mathrm{~mL}$ of ammonium acetate buffer ( $\mathrm{pH} 4.5), 1 \mathrm{~mL}$ of hydroxylamine hydrochloride (HAH) solution, and $2 \mathrm{~mL}$ of $p$-toluenesulfonic acid, and then followed by adding $25 \mathrm{~mL}$ of acetonitrile and vortexing for $4 \mathrm{~min}$. The samples were added with $5 \mathrm{~g}$ of alumina, shook vigorously and centrifuged for $15 \mathrm{~min}$ at $4000 \mathrm{rpm}$. The supernatant of sample was decanted into $250 \mathrm{~mL}$-separate funnel containing $50 \mathrm{~mL}$ of water and $2 \mathrm{~mL}$ of diethylene glycol. Sample was re-extracted with an additional $25 \mathrm{~mL}$ of acetonitrile, vortexing for $4 \mathrm{~min}$, and centrifuged for $15 \mathrm{~min}$. The supernatant was combined to the first extract in the separatory funnel. $25 \mathrm{~mL}$ of dichloromethane was added into the funnel and shook for $3 \mathrm{~min}$. The lower dichloromethane layer was collected to the end point tube. The upper aqueous layer was re-extracted with $25 \mathrm{~mL}$ of dichloromethane. The lower layer was combined to the first liquid extract. The extract was concentrated to $c a$. $10 \mathrm{~mL}$ under $\mathrm{N}_{2}$ gas. The concentrated extract was moved to a centrifuge tube containing an excess amount of $10 \mathrm{~N} \mathrm{NaOH}$ for defatting by saponification. It was allowed to form the emulsion layer. For clear separation of the organic layer from the emulsion, it may be centrifuged for $5 \mathrm{~min}$. After centrifugation, the upper layer was loaded to a solid phase extraction (SPE) cartridge composite, which consisted of an alumina (ALN)-SPE cartridge (Waters, Co., Milford, MA, USA) connected to the top of a propylsulfonic acid (PRS)SPE cartridge (Varian Co., Lake Forest, CA, USA) using an adapter and conditioned with $5 \mathrm{~mL}$ of methanol and acetonitrile. After sample loading, it was eluted under vacuum with a flow rate of approximately $4 \mathrm{~mL} / \mathrm{min}$. The tube was then rinsed with $5 \mathrm{~mL}$ of acetonitrile twice. After all of the solvent was eluted, the ALN-SPE cartridge was removed from the PRS-SPE cartridge. The PRS-SPE cartridge was washed with $5 \mathrm{~mL}$ of acetonitrile and eluted by gravity into the end point tube with $4 \mathrm{~mL}$ of mobile phase A (ammonium acetate buffer: ACN 50:50). The eluent was concentrated to $1 \mathrm{~mL}$ under $\mathrm{N}_{2}$ gas. The simple flow chart for sample preparation is presented in Scheme 2.

LC/MS analysis. The LC/MS consists of an Agilent Technologies 1100 Series LC system (Palo Alto, CA, USA) interfaced to a Thermo Electron TSQ Quantum mass spectrometry (San Jose, CA, USA). An LC column was a Prodigy ODS-3 column (C18, $5 \mu \mathrm{m}, 4.6 \times 250 \mathrm{~mm}$, Phenomenex Co., Torrance, CA, USA) with a guard column of the same phase. LC separation was operated by gradient with a mobile phase A $(50 \mathrm{mM}$ ammonium acetate (pH 4.5): ACN 50:50) and a mobile phase 


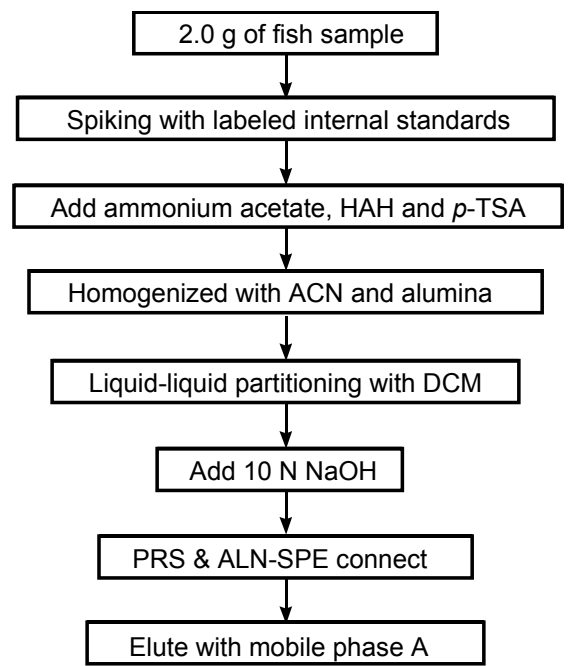

$\mathrm{HAH}$ : hydroxylamine hydrochloride

p-TSA: $p$-toluen sulfonic acid

ACN: acetonitrile

DCM: dichloromethane

PRS \& ALN-SPE: propylsulfonic acid and alumina SPE composite

Mobile phase A: ammonium acetate $(50 \mathrm{mM}, \mathrm{pH} 4.5)$ : acetonitrile (50:50)

Scheme 2. Flow chart of sample preparation and clean-up process.

$\mathrm{B}(\mathrm{ACN})$ at a flow rate of $1.0 \mathrm{~mL} / \mathrm{min}$. The gradient started at $100 \% \mathrm{~A}$ for $5 \mathrm{~min}$ and has a linear gradient to $50 \% \mathrm{~B}$ for $5 \mathrm{~min}$ and $100 \% \mathrm{~B}$ for another $5 \mathrm{~min}$. After then, 100\% B was kept isocratic for $10 \mathrm{~min}$ and changed to the initial mobile phase for the next run. The mass spectrum was obtained in positive mode at the optimized spray conditions. The MS was operated in a selected ion monitoring (SIM) mode to detect $[\mathrm{M}]^{+}$ions of $\mathrm{MG}$ and $d_{5}-\mathrm{MG}$ at $\mathrm{m} / z 329$ and 334 and $[\mathrm{M}+\mathrm{H}]^{+}$ions of LMG and ${ }^{13} \mathrm{C}_{6}$-LMG at $m / z 331$ and 337, respectively.

\section{Results and Discussion}

LC/MS of MG and LMG. The MG has a positive charge itself, thus it was detected as a $[\mathrm{M}]^{+}$ion at $m / z 329$, while the LMG was obtained as a protonated form $[\mathrm{M}+\mathrm{H}]^{+}$at $\mathrm{m} / z 331$ in full-scan mass spectra as shown in Figure 1. The reconstructed ion chromatograms of MG, LMG, $d_{5}$-MG and ${ }^{13} \mathrm{C}_{6}$-LMG in isotope ratio standard (a) and in fortified fish (b) are shown in Figure 2 at $m / z$ 329, 331, 334, and 337, respectively. MG and $d_{5}$-MG were eluted at $6.3 \mathrm{~min}$ and $\mathrm{LMG}$ and ${ }^{13} \mathrm{C}_{6}$-LMG at 13.6 min. MG and LMG are air and light sensitive compounds. For their stability, MG standard solution was prepared with $1 \%$ acetic acid and LMG standard solution with $10 \mu \mathrm{g} / \mathrm{mL}$ ascorbic acid, separately. Hajee et al reported that ascorbic acid was added in LMG standard solution to prevent its photo-oxidative demethylation. ${ }^{33}$ Goldacre et al. reported that slow precipitation of MG by transforming to carbinol can be avoided at acidic condition. ${ }^{34}$ We observed that addition of acetic acid was much effective on the stabilization of MG in standard solution than that of ascorbic acid. However, acetic acid caused LMG to be very unstable. Therefore, standard solutions were prepared separately for MG and LMG. Stock solutions of MG and LMG $(2 \mathrm{mg} / \mathrm{kg})$ were stored in the dark at $4{ }^{\circ} \mathrm{C}$ and pre-

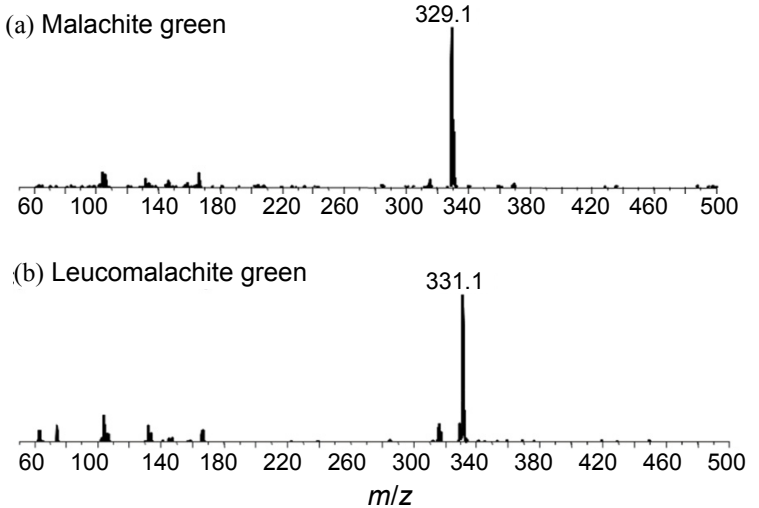

Figure 1. Mass spectra of (a) $1 \mathrm{mg} / \mathrm{kg}$ malachite green in acetonitrile with $1 \%$ acetic acid and (b) $1 \mathrm{mg} / \mathrm{kg}$ leucomalachite green in acetonitrile. (a) Isotope ratio standard $(5 \mu \mathrm{g} / \mathrm{kg})$

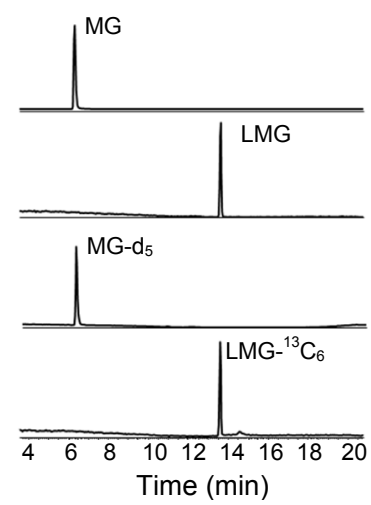

(b) Fortified fish $(5 \mu \mathrm{g} / \mathrm{kg})$

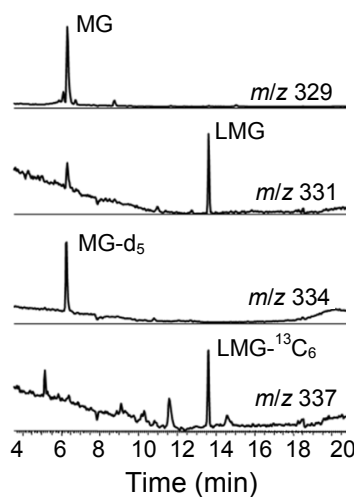

Figure 2. Typical LC/MS selected ion chromatogram of malachite green, leucomalachite green, $\mathrm{d}_{5}$-malachite green and ${ }^{13} \mathrm{C}_{6}$-leucomalachite green in isotope ratio standard (a) and in fortified fish (b). Malachite green and $\mathrm{d}_{5}$-malachite green was eluted at $6.3 \mathrm{~min}$ and leucomalachite green and ${ }^{13} \mathrm{C}_{6}$-leucomalachite green at $13.6 \mathrm{~min}$.

Table 1. Limits of detection (LOD) and limits of quantification (LOQ) for MG and LMG by the ID-LC/MS method

\begin{tabular}{ccc}
\hline & LOD $(\mathrm{S} / \mathrm{N}: 3)(\mu \mathrm{g} / \mathrm{kg})$ & LOQ $(\mathrm{S} / \mathrm{N}: 10)(\mu \mathrm{g} / \mathrm{kg})$ \\
\hline MG & 0.02 & 0.08 \\
LMG & 0.02 & 0.08 \\
\hline
\end{tabular}

pared every two weeks. Working solutions were prepared by diluting with the same solvents as those for the stock solutions at the beginning of each set of analysis.

Table 1 lists the limits of detection and quantification of MG and LMG with the LC/MS method. The detection limits for the LC/MS method were $0.02 \mu \mathrm{g} / \mathrm{kg}$ for both MG and LMG with signal to noise ratio of 3 in the SIM mode. The limits of quantification for fortified salmon sample were $0.08 \mu \mathrm{g} / \mathrm{kg}$ for both MG and LMG with signal to noise ratio of 10 using the SIM mode.

In this study, the method using a selected reaction monitoring (SRM) mode was studied; however, at the lower concentration for the incurred sample, the relative standard deviation of 
Table 2. The comparison of recoveries for the fortified salmon tissue $(10 \mu \mathrm{g} / \mathrm{kg})$ with and without the saponification step during sample preparation process

\begin{tabular}{cccccc}
\hline & \multicolumn{2}{c}{ With saponification } & & \multicolumn{2}{c}{ Without saponification } \\
\cline { 2 - 3 } \cline { 5 - 6 } & MG & LMG & & MG & LMG \\
\hline Sample 1 & $81 \%$ & $61 \%$ & & $9 \%$ & $13 \%$ \\
Sample 2 & $73 \%$ & $45 \%$ & & $16 \%$ & $1 \%$ \\
Sample 3 & $51 \%$ & $69 \%$ & & $14 \%$ & $6 \%$ \\
\hline
\end{tabular}

Table 3. The comparison of recoveries for fortified tuna tissues $(50 \mu \mathrm{g} /$ $\mathrm{kg}$ ) with and without the saponification step during sample preparation process

\begin{tabular}{ccccccc}
\hline & \multicolumn{2}{c}{ With saponification } & & \multicolumn{2}{c}{ Without saponification } \\
\cline { 2 - 3 } \cline { 5 - 6 } & MG & LMG & & MG & LMG \\
\hline Sample 1 & $61 \%$ & $47 \%$ & & $18 \%$ & $2 \%$ \\
Sample 2 & $50 \%$ & $58 \%$ & & $14 \%$ & $1 \%$ \\
Sample 3 & $52 \%$ & $67 \%$ & & $17 \%$ & $10 \%$ \\
\hline
\end{tabular}

run-to-run isotope ratio measurements was much larger than that with SIM mode. Thus, results were obtained by SIM mode.

Sample preparation step for defatting. In our laboratory, the salmon was selected for the blank fish because the provided incurred fish was salmon tissue. The blank salmon from the local market was tested by the LC/MS method and the results showed that the level of MG and LMG was below the detection limit of LC/MS conditions. However, following the sample preparation procedures in the literature ${ }^{27}$ resulted in seriously low recoveries of MG and LMG from salmon tissue. The recovery of $\mathrm{MG}$ and $\mathrm{LMG}$ with this method was below $15 \%$ as shown in Table 2 . The reason of low recovery could be demonstrated that the substantial amounts of fat extracted from fish tissue saturated the Alumina and PRS-SPE column in sample preparation, thus MG and LMG were not retained and just eluted through the SPE column. To solve this problem, the procedure was modified with the additional saponification step before loading sample extract to the SPE column. A $10 \mathrm{~N} \mathrm{NaOH}$ solution was used for saponification of fat from the salmon tissue extract. After addition of $\mathrm{NaOH}$ solution, the extract showed an emulsion layer between the organic layer and the aqueous layer. This layered solution was centrifuged to shrink the emulsion layer, after then, the upper organic layer was taken using a syringe. This extract was loaded to the SPE column. Table 2 showed that the modified procedure enhanced the recoveries of MG and LMG dramatically. While the recoveries of MG and LMG from salmon tissue without saponification was below $15 \%$, the recoveries with saponification were $50 \sim 80 \%$. To confirm the relationship between recovery and saponification, the experiment was performed with tuna using the modified procedure. When the $\mathrm{NaOH}$ solution was added to the extract of tuna sample, the emulsion layer was formed, but it was thinner than that from the salmon extract. As shown in Table 3, the saponification step also improved recoveries of MG and LMG from tuna. The effect of saponification was examined for salmon samples fortified with MG and LMG to 1,5 and $20 \mu \mathrm{g} / \mathrm{kg}$.
Table 4. Recoveries for MG and LMG from salmon tissue at various levels of fortifying concentration with saponification step

\begin{tabular}{cccc}
\hline \multirow{3}{*}{$1 \mu \mathrm{g} / \mathrm{kg}$} & & MG & LMG \\
\hline & Sample 1 & $58 \%$ & $54 \%$ \\
& Sample 2 & $52 \%$ & $62 \%$ \\
& Sample 3 & $72 \%$ & $80 \%$ \\
\hline \multirow{3}{*}{$5 \mu \mathrm{g} / \mathrm{kg}$} & Sample 1 & $56 \%$ & $48 \%$ \\
& Sample 2 & $67 \%$ & $54 \%$ \\
& Sample 3 & $55 \%$ & $63 \%$ \\
\hline \multirow{2}{*}{$20 \mu \mathrm{g} / \mathrm{kg}$} & Sample 1 & $70 \%$ & $52 \%$ \\
& Sample 2 & $79 \%$ & $50 \%$ \\
& Sample 3 & $57 \%$ & $69 \%$ \\
\hline
\end{tabular}

Table 5. Measurement results for fortified salmon tissue at various levels using ID-LC/MS

\begin{tabular}{lccccc}
\hline & \multicolumn{2}{c}{${\text { Fortified level }(\mu \mathrm{g} / \mathrm{kg})^{a}}$} & & \multicolumn{2}{c}{ Measured level $(\mu \mathrm{g} / \mathrm{kg})^{b}$} \\
\cline { 2 - 3 } \cline { 5 - 6 } & MG & LMG & & MG & LMG \\
\hline Sample 1 & 0.8 & 0.8 & & $0.79 \pm 0.03$ & $0.81 \pm 0.04$ \\
Sample 2 & 0.8 & 0.8 & & $0.78 \pm 0.03$ & $0.81 \pm 0.04$ \\
Sample 3 & 4.2 & 4.0 & & $4.02 \pm 0.06$ & $4.11 \pm 0.19$ \\
Sample 4 & 4.2 & 4.0 & & $4.28 \pm 0.06$ & $4.04 \pm 0.09$ \\
Sample 5 & 18.7 & 18.8 & & $18.50 \pm 0.37$ & $18.71 \pm 1.29$ \\
Sample 6 & 18.0 & 18.0 & & $17.23 \pm 0.36$ & $17.06 \pm 0.50$ \\
\hline
\end{tabular}

$a_{\text {the uncertainty of the gravimetrically fortified level is rather minimal com- }}$ pared to the uncertainty in analytical measurement, thus is not reported in this table. ${ }^{b}$ The value following " \pm " is the expanded uncertainty with a level of confidence of $95 \%$.

The results were shown in Table 4 . The recoveries ranged from $50 \%$ to $79 \%$ for $M G$ and from $45 \%$ to $80 \%$ for LMG.

Validation of the method and analytical quality check. We carefully evaluated whether the preparation procedure with saponification and ID-LC/MS in SIM mode has adequate quality. Salmon tissue samples fortified with MG and LMG at various levels $(0.8,4.0$, and $18.0 \mu \mathrm{g} / \mathrm{kg})$ were analyzed by the ID-LC/MS method. The results are listed in Table 5. Measurement results of the fortified samples by the ID-LC/MS method agreed with the gravimetrically fortified value within their uncertainties. That means the sample preparation procedure with saponification and the ID-LC/MS method are appropriate for the analysis of $\mathrm{MG}$ and $\mathrm{LMG}$ residues in fish.

Another way to validate the method is using a certified reference material (CRM). However, to our knowledge, there was no available CRM for MG or LMG in food matrix. We obtained the salmon tissue incurred with MG from Laboratory of Government Chemistry (UK) as a study sample for an interlaboratory comparison (CCQM-P88) among national metrological institutes (NMIs), which was organized by Consultative Committee for Amount of Substance- Metrology in Chemistry (CCQM). The final levels of MG and LMG and homogeneity test of material were assessed by LGC. Two pots of incurred material were obtained and three of subsamples from each pot were analyzed by the ID-LC/MS method. Our results and the provided values from LGC are listed in Table 6 . The values with the proposed method were $2.03 \pm 0.16 \mu \mathrm{g} / \mathrm{kg}$ for $\mathrm{MG}$ and $6.94 \pm 0.54 \mu \mathrm{g} / \mathrm{kg}$ for LMG. The reference values pro- 
Table 6. Results from analysis of the reference material by LGC based on ID-LC/MS

\begin{tabular}{ccc}
\hline & MG $(\mu \mathrm{g} / \mathrm{kg})$ & LMG $(\mu \mathrm{g} / \mathrm{kg})$ \\
\hline Sample 1-1 & 2.10 & 6.52 \\
Sample 1-2 & 2.13 & 6.47 \\
Sample 1-3 & 2.09 & 6.68 \\
Sample 2-1 & 2.12 & 6.67 \\
Sample 2-2 & 1.82 & 7.64 \\
Sample 2-3 & 1.95 & 7.68 \\
\hline Average & $2.03 \pm 0.16^{a}$ & $6.94 \pm 0.54^{a}$ \\
LGC & $2.52 \pm 0.36^{a}$ & $6.80 \pm 0.86^{a}$
\end{tabular}

${ }^{a}$ The value following " \pm " is the expanded uncertainty with a level of confidence of $95 \%$.

vided by $L G C$ were $2.52 \pm 0.36 \mu \mathrm{g} / \mathrm{kg}$ for $\mathrm{MG}$ and $6.80 \pm 0.86$ $\mu \mathrm{g} / \mathrm{kg}$ for LMG. ${ }^{35}$ The results were in good agreement within their uncertainties. However, the uncertainties of LGC value are about twice as much as that of our value for both $\mathrm{MG}$ and LMG. That means our proposed method much improves the accuracy of determination for MG and LMG in fish.

\section{Conclusions}

The accurate and sensitive ID-LC/MS method for the quantification of $\mathrm{MG}$ and $\mathrm{LMG}$ residues in fish has been presented in this study. Each isotope solutions were spiked to the sample fish for quantification of MG and LMG, respectively, by IDLC/MS. In addition, the new sample preparation method which employs the saponification step to remove fat in sample extracts improved the overall recoveries of MG and LMG. This was evaluated with gravimetrically fortified salmon and tuna. Comparison with the value of the reference material proved that the proposed method is appropriate and much improved accuracy for the quantification of MG and LMG residues in fish.

\section{References}

1. Foster, F. J.; Woodbury, L. Prog. Fish Cult. 1936, $18,7$.

2. Alderman, D. J. Fish Dis. 1985, 8, 289.

3. Swarbrick, A.; Murby, E. J.; Hume, P. J. Liq. Chrom. Rel. Technol. 1997, 20, 2269.

4. Doerge, D. R.; Chang, H. C.; Divi, R. L.; Churchwell, M. I. Chem. Res. Toxicol. 1998, 11, 1098.

5. Fessard, V. T.; Godard, S.; Huet, A.; Mourot, J. M. J. Appl. Toxicol. 1999, 19, 421
6. Littlefield, J. D.; Blackwell, B.-N.; Hewitt, C. C.; Gaylor, D. W. Fundam. Appl. Toxicol. 1985, 5, 902.

7. McDonald, J. J.; Cerniglia, C. E. Drug Metab. Dispos. 1984, 12, 330.

8. Commission Decision of 22 Dec., 2003 amending Decision 2002/ $657 / \mathrm{EC}$ as regards the setting of minimum required performance limits (MRPLs) for certain residues in food of animal origin, O.J. Europ. Comm. L 6, pp 38-39.

9. Culp, S. J.; Blankenship, L. R.; Kusewitt, D. F.; Doerge, D. R.; Mulligan, L. T.; Beland, F. A. Chemico-Biological Interactions 1999, 122, 153.

10. Culp, S. G.; Beland, F. A. J. Am. Coll. Toxicol. 1996, 15, 219.

11. Allen, J. L.; Gofus, J. E.; Meinertz, J. R. J. Assoc. Off. Anal. Chem. Int. 1994, 77, 553.

12. Rushing, L. G., Webb, S. F.; Thompson, H. C., Jr. J. Chromatogr. $B$ 1995, 674, 125.

13. Roybal, J. E.; Penning, A. P.; Munns, R. K.; Holland, D. C.; Hurlbut, J. A.; Long, A. R. J. of AOAC Int. 1995, 78, 453.

14. Valle, L.; Diaz, C.; Zanocco, A. L.; Tichter, P. J. Chromatogr A 2005, 1067, 101

15. Bergwerff, A. A.; Scherpeniss, P. J. Chromatogr. B 2003, 788, 351.

16. Wu, X.; Zhang, G. Y.; Hou, Wu. X.; Yuan, Z. J. Chromatogr. A 2007, 1172, 121.

17. Doerge, D. R.; Churchwell, M. I.; Gehring, T. A.; Pu, Y. M.; Plakas, S. M. Rapid Commun. Mass Spectrom. 1998, 12, 1625.

18. Dowling, G.; Mulder, P. P. J.; Duffy, C.; Regan, G.; Smyth, M. R. Anal. Chimi. Acta 2007, 586, 411.

19. Valle, L.; Diaz, C.; Zanocco, A. L.; Richter, P. J. Chromatogr. A 2005, 1067, 101 .

20. Allen, J. L.; Meinertz, J. R. J. Chrom. 1991, 536, 217.

21. Turnipseed, S. B.; Roybal, J. E.; Hurlbut, J. A.; Long, A. R. J. Assoc. Off. Anal. Chem. Int. 1995, 78, 971.

22. Rushing, L. G. J. Chromatogr. B 1997, 700, 223.

23. Alderman, D. J.; Hadley, R. S. J. Fish Dis. 1993, 16, 297.

24. Mitrowska, K.; Posyniak, A.; Zmudzki, J. J. Chromatogr. A 2005, 1089, 187.

25. Eng, G. Y.; Maxwell, R. J.; Cohen, E.; Piotrowski, E. G.; Fiddler, W. J. Chromatogr. A 1998, 799, 349.

26. Perera, C.; Hoover, R. Food Chem. 1999, 64, 361

27. Seo, J.; Kim, H.-Y.; Chung, B.C.; Hong, J. J. Chromatgr. A 2005, 1067, 303.

28. Berdie, L.; Grimait, J. O. J. Chromatogr. A 1998, 823, 373.

29. Jung, P. G.; Kim, B.; Park, S.-R.; So, H.-Y.; Shi, L.-H.; Kim Y. Anal. Bioanal. Chem. 2004, 380, 782.

30. Park, S.; Kim, B.; So, H.-Y.; Kim, Y.-J.; Kim, J. J. Bull Korean Chem. Soc. 2007, 28, 737 .

31. Doerge, D. R.; Chang, H. C.; Divi, R. L.; Churchwell, M. I. Chem. Res. Toxicol. 1998, 11, 1098 .

32 Anderson, W. C.; Roybal, J. E.; Turnipseed, S. B. J. AOAC Int. 2005, 88, 1292.

33. Hajee, C. A. J.; Haagsma, N. J. Chromatogr. B 1995, 669, 219.

34. Goldacre, R. J.; Phillips J. N. J. Chem. Soc. 1949, 1724.

35. Hall, Z.; Hopley, C.; O'Connor, G. J. Chromatogr. B 2008, 874, 95. 